\title{
Multiscale anisotropic diffusion for ringing artifact suppression in geophysical deconvolution data
}

\author{
Boxin Zuo $\cdot$ Xiangyun Hu $\cdot$ Meixia Geng
}

Received: 21 September 2015/ Accepted: 6 July 2016/Published online: 19 August 2016

(C) The Author(s) 2016. This article is published with open access at Springerlink.com

\begin{abstract}
Ringing artifact degradations always appear in the deconvolution of geophysical data. To address this problem, we propose a postprocessing approach to suppress ringing artifacts that uses a novel anisotropic diffusion based on a stationary wavelet transform (SWT) algorithm. In this paper, we discuss the ringing artifact suppression problem and analyze the characteristics of the deconvolution ringing artifact. The deconvolution data containing ringing artifacts are decomposed into different SWT subbands for analysis, and a new multiscale adaptive anisotropic filter is developed to suppress these degradations. Finally, we demonstrate the performance of the proposed method and describe the experiments in detail.
\end{abstract}

Keywords Deconvolution · Ringing artifacts · Anisotropic diffusion $\cdot$ Stationary wavelet transform algorithm .

Multiscale

\section{Introduction}

Deconvolution algorithms have been used to process data from a wide range of geophysical techniques, including for gravity, magnetic, and seismic data. Zuo and $\mathrm{Hu}$ (2012) also introduced a deconvolution algorithm for processing magnetotelluric data. However, the ringing artifacts produced by these deconvolution algorithms are rarely reported. Most of the research on ringing artifacts focuses on the enhancement of image compression techniques (Perona and Malik 1990; Weickert 1998; Pollak et al. 2000; Lysaker et al. 2003; Ding et al. 2005). Popovici and

B. Zuo $\cdot$ X. Hu $(\bowtie) \cdot$ M. Geng

China University of Geosciences, Wuhan 430074, China

e-mail: xyhu@163.com
Withers (2007) proposed using a bilateral filter to suppress ringing artifacts from compressed images. The discrete wavelet transform algorithm was first used by Kim et al. (2009) to analyze ringing artifacts in multilevel edge maps for compressed images. These algorithms perform well for the suppression of ringing artifacts in compressed images, but are not suitable for processing deconvolution data from geophysical measurements.

The ringing artifacts that occur in the deconvolution of geophysical data are not the same as the artifacts in compressed images. In general, the ringing artifacts in deconvolution algorithms present as stronger oscillations than the artifacts in compressed images (Fig. 1).

The dotted lines L1 and L3 represent the same data selected from one row of observational data. Line L2 is the result of compressing L1 using the JPEG 2000 coded algorithm, while L4 is the result of deconvoluting L3.

Geophysical deconvolution algorithms introduce ringing artifacts as a result of errors in the observational data, and they commonly generate intense oscillations in areas of data discontinuity, e.g., edges and noise. They also often appear as false anomalous edges. The characteristics of the ringing artifacts seen after the deconvolution of geophysical data are very different from those of the ringing artifacts in compressed images. Therefore, it is not possible to directly suppress the ringing artifacts resulting from the deconvolution of geophysical data using traditional diffusion algorithms for compression images. Based on this analysis, we propose a novel method to suppress ringing artifacts arising from the deconvolution of geophysical data.

The discrete wavelet transform is a valuable signal analysis tool and can highlight specific subband features (Arivazhagan et al. 2007) through the decomposition of 


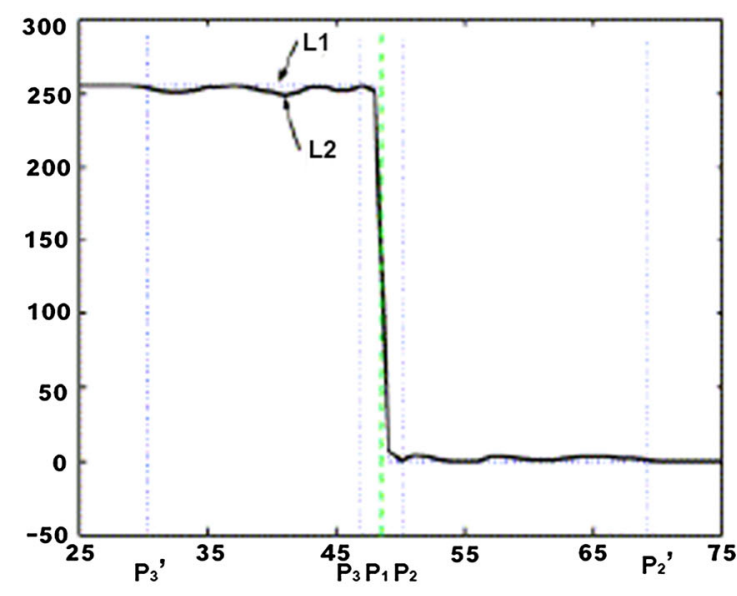

(a)

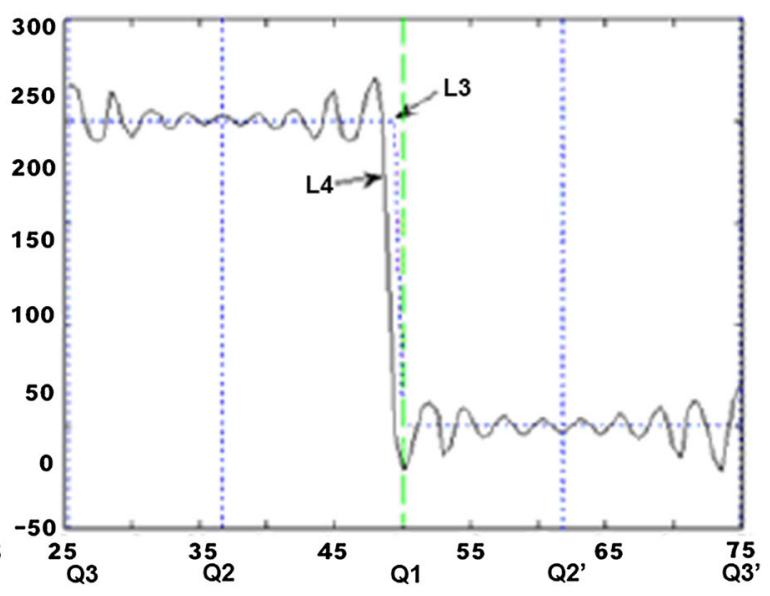

(b)

Fig. 1 a Ringing artifacts in a compressed image (Marziliano and Dufaux 2004). b Ringing artifacts produced during deconvolution of geophysical data (Zuo et al. 2009)

multiple scalars in complex data. Discrete wavelet transform is commonly applied for edge detections and contrast enhancements in various applications (Huang et al. 2002; Nakashizuka et al. 2004; Hatami et al. 2005). Pesquet et al. (1996) suggested using the stationary wavelet transform (SWT) algorithm to make the subband coefficients time invariant and to improve its power. In the SWT method, the coefficients have the same length as the original data at every level. It uses a stationary transform instead of traditional wavelet downsampling by adding an upsampling procedure on every scale coefficient (Gao et al. 2008). The redundancy of the SWT algorithm facilitates the detection of weak anomalies in the data, especially the detection of the false edges of ringing artifacts.

A nonlinear anisotropic diffusion method was proposed by Perona and Malik (1990) and has been widely studied in a number of scientific fields over the past two decades (Black et al. 1998; Pollak et al. 2000; Chen and Hsu 2005; Ding et al. 2005). The advantage of this method is that it can adaptively smooth noise while simultaneously preserving the actual model boundaries. Therefore, we used it to analyze ringing artifacts in the decomposed subbands of the SWT algorithm. In summary, we propose an anisotropic diffusion method to suppress the ringing artifacts in the results of the deconvolution of geophysical data based on SWT analysis.

The background to the SWT algorithm is briefly reviewed and then the proposed ringing artifact anisotropic filter based on the SWT algorithm is discussed. We describe our proposed multiresolution system and then present our experimental results and discussion before providing conclusions on our work.

\section{Background to the SWT algorithm}

Assuming $H$ and $G$ are the coefficients of the wavelet filters, then $H^{[j]}\left(H^{[0]}=H, j=1,2, \ldots, n\right)$ and $G^{[j]}\left(G^{[0]}=G, j=1\right.$, $2, \ldots, n)$ can be obtained by inserting a zero between every adjacent pair of elements. If $a_{0}$ is an initial signal sequence, the signal stationary wavelet (Pesquet et al. 1996) is

$\left\{\begin{array}{l}a_{j+1}=H^{[j]} a_{j} \\ b_{j+1}=G^{[j]} b_{j}\end{array}\right.$,

where $a_{j+1}$ represents the approximation coefficient of the SWT algorithm and $b_{j+1}$ is the detail. We used $a_{j}\left(\varepsilon_{1}, \ldots, \varepsilon_{j}\right)$ and $b_{j}\left(\varepsilon_{1}, \ldots, \varepsilon_{j}\right)$ to denote the coefficients obtained from the SWT algorithm at level $j$

$\phi_{j}\left(a_{j+1}, b_{j+1}\right)=H^{*} a_{j+1}\left(\varepsilon_{j+1}\right)+G^{*} b_{j+1}\left(\varepsilon_{j+1}\right),\left(\varepsilon_{j+1}=0\right)$,

$\varphi_{j}\left(a_{j+1}, b_{j+1}\right)=H^{*} a_{j+1}\left(\varepsilon_{j+1}\right)+G^{*} b_{j+1}\left(\varepsilon_{j+1}\right),\left(\varepsilon_{j+1}=1\right)$,

where * represents a conjugate operator. The inverse SWT is

$a_{j}\left(\varepsilon_{j}\right)=\frac{1}{2}\left[\phi_{j}\left(a_{j+1}, b_{j+1}\right)+\varphi_{j}\left(a_{j+1}, b_{j+1}\right)\right]$.

\section{Ringing artifact anisotropic diffusion filter based on the SWT algorithm}

The basic mechanism behind our approach can be analyzed by comparing the detailed SWT coefficients of the deconvolution results with the observed ringing artifacts (Fig. 2). $L L_{2}$ represents the output from the low-pass filter in the horizontal and vertical directions, whereas $H L_{i}$ are 


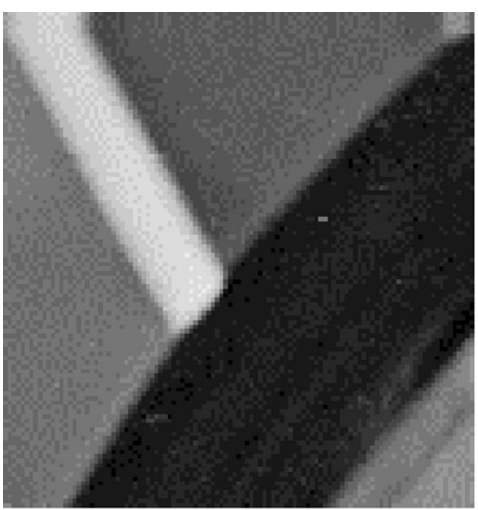

(a)

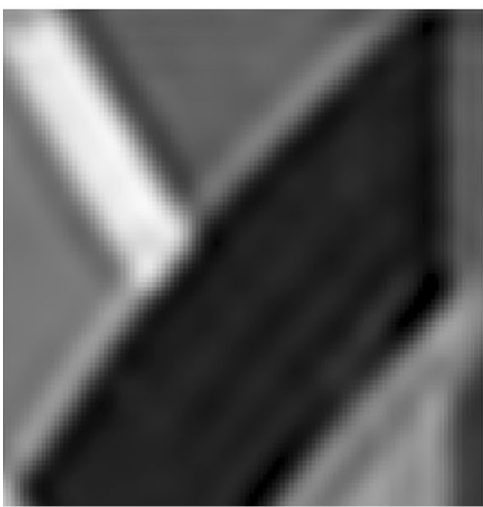

(d)

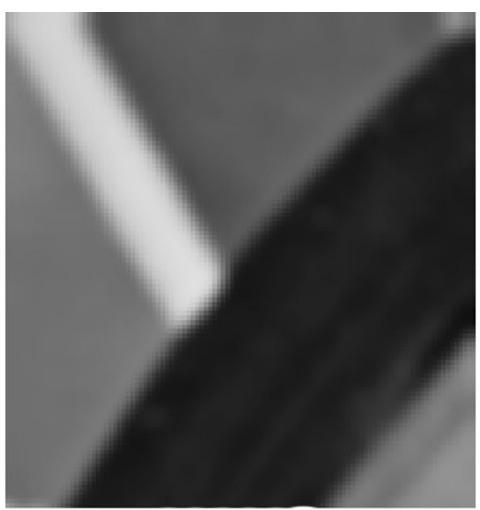

(b)

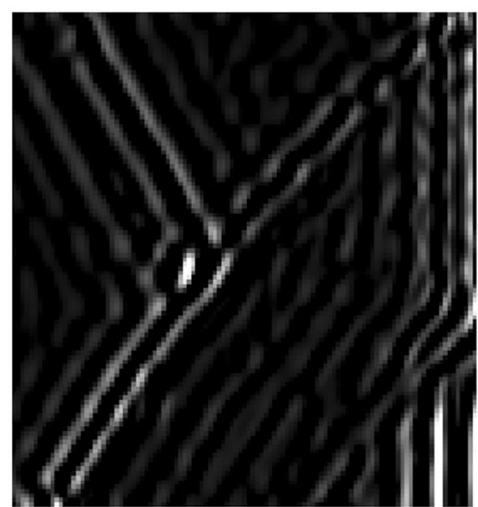

(e)

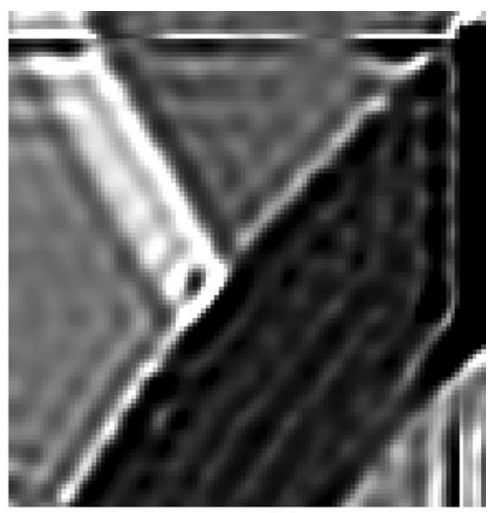

(c)

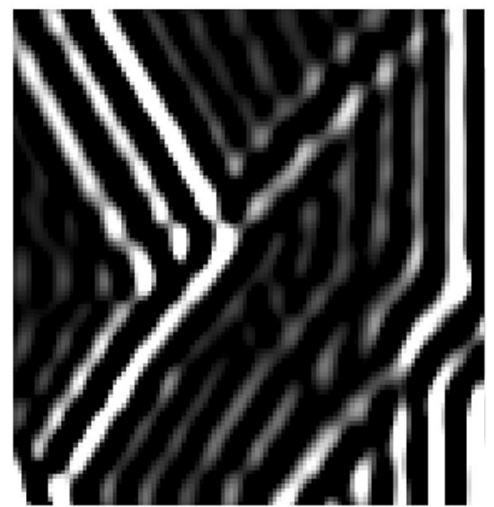

(f)

Fig. 2 a Actual model. b Observation data. c Deconvolution results showing ringing artifacts. d Low-frequency wavelet coefficients $L L_{2}$. e High-frequency wavelet coefficients $H L_{1}$. f High-frequency wavelet coefficients $H L_{2}$

the outputs from the high-pass filter in the vertical and horizontal directions. All the high-frequency wavelet coefficients are magnified by a coefficient of 2 in this paper so that they can be more easily seen.

In Fig. 2d, the low-frequency wavelet coefficient $L L_{2}$ does not appear in any of the ringing artifacts, so it does not need to be filtered. However, Fig. 2e, f shows high levels of ringing artifacts in the decomposed high-frequency coefficients. In addition, the contrast in these coefficients increases as the level of decomposition $k$ increases. A new anisotropic filter is suggested by this analysis, which works on the decomposed SWT coefficients as follows.

For a high-frequency wavelet coefficient $H_{k}(x, y)$ decomposed by the SWT algorithm and with a finite power and no zero values over the data support value $\Omega, k$ is the level of decomposition of the SWT algorithm $k(k=0,1,2, \ldots, n)$. The output $H_{k}(x, y, t)$ can be expressed according to the following partial differential equation:

$\left\{\begin{array}{l}\frac{\partial}{\partial t} H_{k}(x, y, t)=\nabla \cdot\left[c(q) \nabla H_{k}(x, y, t)\right] \\ H_{k}(x, y, 0)=H_{k}(x, y),\left.\left(\partial H_{k}(x, y, t) / \partial \vec{n}\right)\right|_{\partial \Omega}=0\end{array}\right.$,

where $\partial \Omega$ denotes the border of $\Omega$ and $\partial \vec{n}$ is the outer normal of $\partial \Omega$. A new diffusivity function $c(q, k)$ is defined in Eq. (6):

$$
\begin{aligned}
c(q, k)= & (1+\sqrt{k}) /\left\{1+\left[q^{2}(x, y, t, k)-q_{0}^{2}(t, k)\right] /[1\right. \\
& \left.\left.\left.+q_{0}^{2}(t, k)\right)\right]\right\},
\end{aligned}
$$

where $q(x, y, t, k)$ is the instantaneous coefficient determined by

$q(x, y, t, k)=\sqrt{\frac{(1 / 2)\left(\left|\nabla H_{k} / H_{k}\right|\right)^{2}-(1 / 16)\left(\nabla^{2} H_{k} / H_{k}\right)^{2}}{\left[1+(1 / 4)\left(\nabla^{2} H_{k} / H_{k}\right)\right]^{2}}}$.

$q(x, y, t, k)$ serves as the boundary detector for the data. The ringing artifact scale function $q_{0}(t, k)$ is defined to control the degree of smoothing behavior as defined in Eq. (8):

$q_{0}(t, k)=\left(\overline{\operatorname{var}\left(H_{k}(x, y, t)\right)}\right)^{1 / 2} / \overline{\operatorname{mean}\left(H_{k}(x, y, t)\right)}$,

where $\overline{\operatorname{var}(\cdot)}$ and $\overline{\operatorname{mean}(\cdot)}$ are the mean of the intensity variance and the mean of the directions of the variants in the high-frequency wavelet coefficients decomposed by the SWT algorithm over a ringing artifact area, respectively.

The proposed anisotropic filter has some characteristics that are not shared with conventional anisotropic diffusion. In previous research on anisotropic filters, $c(q)$ was equal to a Gaussian filter $K_{\sigma}$ as $K_{\sigma}=\frac{1}{2 \pi \sigma^{2}} \exp \left(-\frac{|x|^{2}+|y|^{2}}{2 \sigma^{2}}\right)$ (Weickert 
1998) and $q_{0}(\cdot)$ was defined as a domain function ( $\mathrm{Yu}$ and Acton 2002). In this paper, Eqs. (6) and (8) define $c(q, k)$, $q(x, y, t, k)$, and $q_{0}(t, k)$ in the wavelet domain without the assumption of a Gaussian distribution. Moreover, $c(q, k)$, $q(x, y, t, k)$, and $q_{0}(t, k)$ are related to the SWT level of decomposition $k$.

As shown in Fig. 2, as the SWT level of decomposition $k$ is increased, the contrast in the ringing artifact increases dramatically. The function $c(q, k)$ then presents a high value and the proposed filter shows higher diffusion behavior as the SWT level of decomposition $k$ is increased.

For a fixed level $k$, according to Eq. (6), the filter shows encouraging anisotropic diffusion in high-contrast edge regions where $q(x, y, t, k)$ is larger than $q_{0}(t, k)$. Otherwise, it acts as an isotropic filter over the ringing and non-edge regions.

Using the proposed anisotropic filter, the filter results for the decomposition of the high-frequency wavelet coefficients in Fig. 2d, f by the SWT algorithm are shown in Fig. 3.

\section{Multiresolution anisotropic diffusion}

The proposed multiresolution ringing artifact anisotropic diffusion method is shown as a flow chart in Fig. 4. First, the deconvolution result is decomposed by the SWT algorithm, then the decomposed coefficients in every detailed subband are filtered by the proposed anisotropic filter. Finally, the inverse SWT algorithm is applied to the filtered results and the final result is the output.

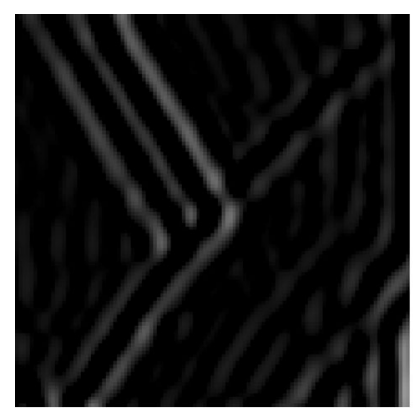

(a)

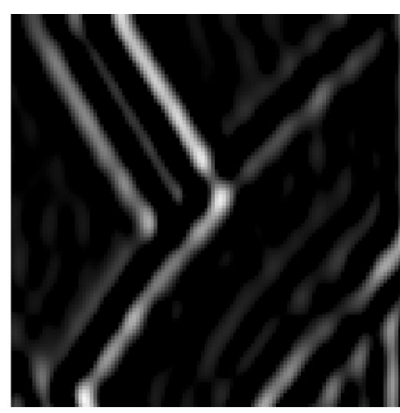

(b)
Fig. 3 a Filtering result for Fig. 2e. b Filtering result for Fig. 2f

\section{Results and discussion}

To test the proposed anisotropic diffusion algorithm, the algorithm was applied to the synthetic model shown in Fig. 2a. The model was fitted by a Gaussian kernel $K=e^{-\left(s_{1}^{2}+s_{2}^{2}\right) /\left(2 \sigma^{2}\right)} / \sum \sum e^{-\left(s_{1}^{2}+s_{2}^{2}\right) /\left(2 \sigma^{2}\right)}$ with a support size $s_{1}=s_{2}=6, \sigma=2$. The observation data are shown in Fig. 2b. A standard deconvolution algorithm was applied to the data with various values of point spread function support size bias $(2,4,6,8)$. The results of using our algorithm for deconvolution and diffusion are shown in Fig. 5.

As Fig. 5a shows, the results of the proposed algorithm give a slightly low-pass effect. This phenomenon is due to the smoothing behavior of anisotropic diffusion. It is also clear that the discontinuities in the data are preserved during the diffusion procedure. Although the numerical range of the geophysical data and the common images are not the same, the characteristics of these ringing artifacts in these data are similar. Commonly, the textures of images are very complex, and its deconvolution result will contain complicated ringing artifact. So we discuss the suppression of the ringing artifact in images in the experiment section as the example. The geophysical data processing is similar to this procedure.

The effectiveness of the algorithm in suppressing ringing artifacts was evaluated by the mean absolute error (MAE) relative to the original model (Fig. 3a), as defined in Eq. (5):

$\mathrm{MAE}=\frac{1}{\mathrm{MN}} \sum_{i=1}^{m} \sum_{j=1}^{m}\left|I(i, j, t)-I_{\text {ori }}\right|$.

The results of the proposed SWT method based on multiresolution anisotropic diffusion are shown in Table 1. By comparing the results with those of the bilateral filter, it is easy to see that the numerical precision of the proposed SWT-based anisotropic diffusion results is higher than that for the bilateral filter, particularly when the ringing artifacts are strong. The proposed diffusion results also have a better visual quality. The bilateral filter results (Fig. 5c) seem to show fewer ringing artifacts and less blur than the proposed diffusion result (Fig. 5b). Based on the numerical statistical results in Table 1 , it is easy to see that the data obtained using conventional anisotropic diffusion (Fig. 5d) are oversmoothed during filtering.

The conventional diffusion and the bilateral filter did not preserve the weak edges. This behavior leads to a decrease

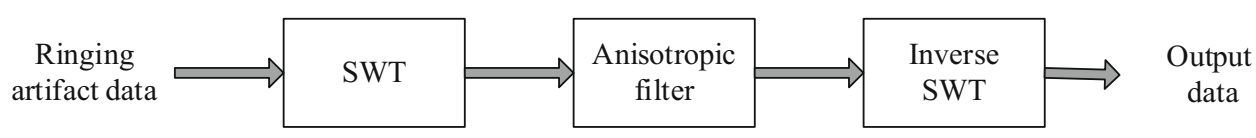

Fig. 4 Flow chart for SWT anisotropic diffusion treatment of ringing artifacts 
(a)

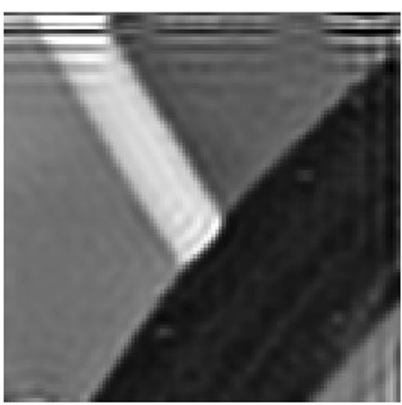

(b)

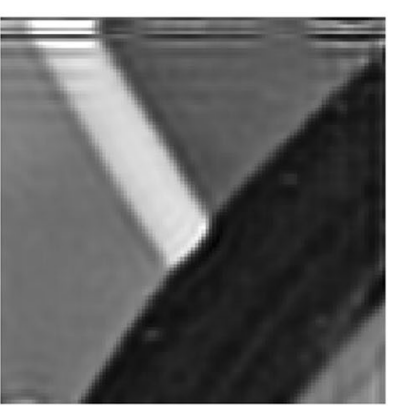

(c)

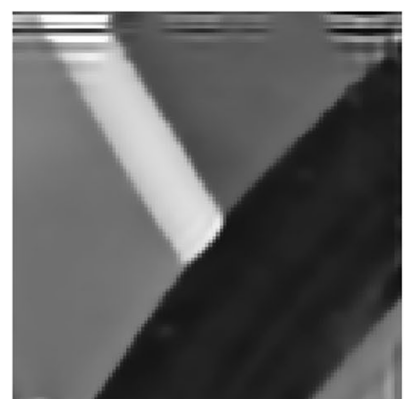

(d)

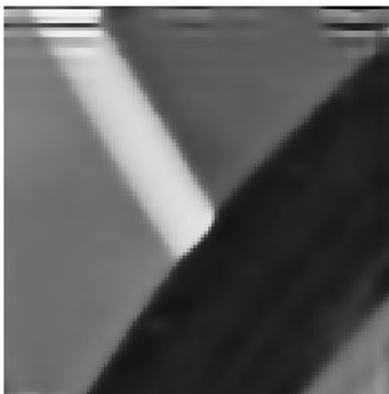

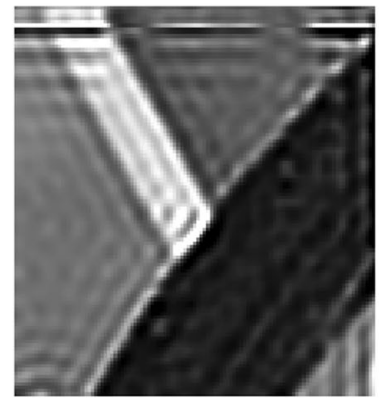
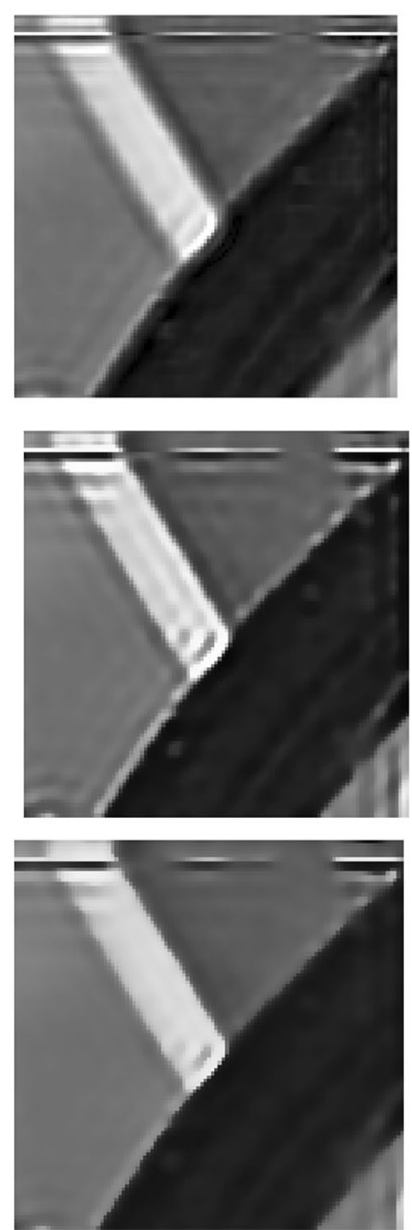
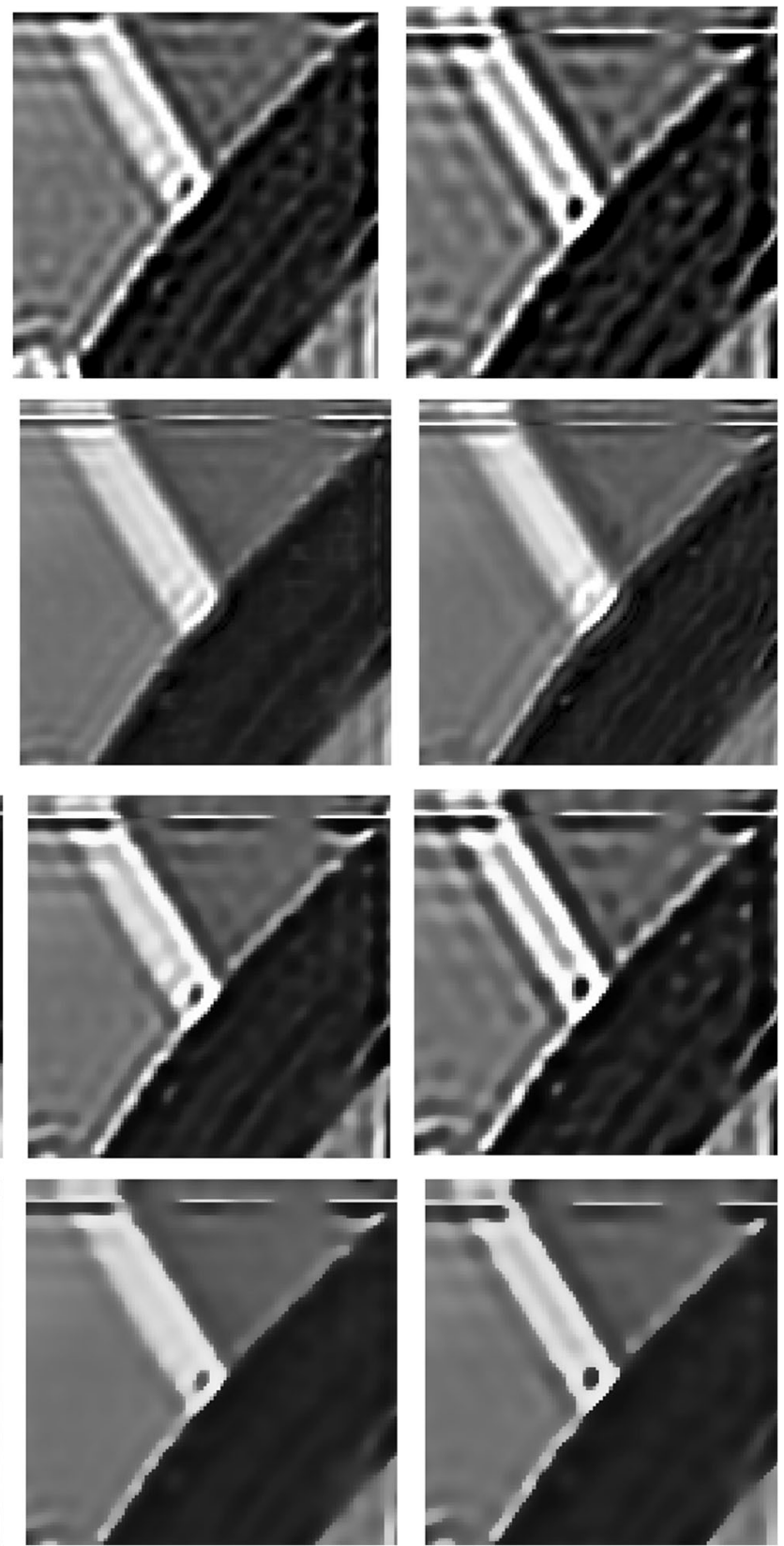

Fig. 5 Diffusion results for deconvolution. Comparison of results obtained by the proposed algorithm with those from other related algorithms. a Deconvolution results obtained with point spread function support size estimated bias values of 2, 4, 6, and 8 , respectively. b Diffusion results obtained by the proposed algorithm. $\mathbf{c}$ Results from a filtered by a bilateral filter. $\mathbf{d}$ Results from a filtered by conventional anisotropic diffusion

in the actual model details, and some false edges appear in the results. In contrast, the proposed method avoids this behavior. Under the conditions of strong ringing artifacts (e.g., Fig. 5a, 4th image), the proposed anisotropic diffusion filter gives a better perceptual quality and numerical precision than the other filters. The MATLAB (version 2014b)-based programs were executed using an Intel i5$2500 \mathrm{k} \mathrm{CPU}, 3.30 \mathrm{GHz}, 8.0 \mathrm{~GB}$ RAM system and the execution time for a $256 \times 256$ size model was $<1$ s.

\section{Conclusions}

This paper has introduced a diffusion algorithm that suppresses ringing artifacts for use in the postprocessing of deconvolution data from geophysical applications. The proposed anisotropic diffusion filter was able to simultaneously suppress the ringing artifacts and preserve the model boundaries. Experiments showed that this algorithm significantly enhanced both the visual and numerical static 
Table 1 Experimental and numerical statistical results

\begin{tabular}{lllcc}
\hline Figure 3a & $\begin{array}{l}\text { Figure 5a } \\
(1 \mathrm{st}-4 \mathrm{th})\end{array}$ & $\begin{array}{l}\text { Figure 5b (1st-4th), } \\
\text { proposed method }\end{array}$ & $\begin{array}{l}\text { Figure 5c (1st-4th), } \\
\text { bilateral filter }\end{array}$ & $\begin{array}{l}\text { Figure 5d (1st-4th), } \\
\text { conventional anisotropic } \\
\text { filter }\end{array}$ \\
\hline Mean absolute error & 0.0735 & 0.0522 & 0.0612 & 0.0578 \\
& 0.1549 & 0.0698 & 0.1245 & 0.0885 \\
& 0.2219 & 0.0749 & 0.1923 & 0.1216 \\
Increasing precision (\%) & 0.1172 & 0.0382 & 0.1063 & 0.0633 \\
& N/A & 28.97 & 16.73 & 21.36 \\
N/A & 54.93 & 19.63 & 42.87 \\
Neak signal-to-noise ratio & N/A & 66.24 & 13.34 & 45.20 \\
& N/A & 67.41 & 9.30 & 45.99 \\
Structural similarity & 24.7306 & 28.5947 & 25.6992 & 26.5445 \\
& 19.5355 & 26.0079 & 20.3793 & 22.5253 \\
& 16.9621 & 25.3502 & 17.5304 & 20.0719 \\
& 15.4078 & 23.3484 & 15.8285 & 18.6624 \\
& 0.6549 & 0.7073 & 0.6737 & 0.6719 \\
& 0.3569 & 0.5804 & 0.4847 & 0.5639 \\
& 0.2272 & 0.5516 & 0.3118 & 0.4578 \\
& 0.1544 & 0.5185 & 0.2046 & 0.3816 \\
\hline
\end{tabular}

performance of the deconvolution results. This algorithm also worked well for the suppression of high-level ringing artifacts.

Open Access This article is distributed under the terms of the Creative Commons Attribution 4.0 International License (http://crea tivecommons.org/licenses/by/4.0/), which permits unrestricted use, distribution, and reproduction in any medium, provided you give appropriate credit to the original author(s) and the source, provide a link to the Creative Commons license, and indicate if changes were made.

\section{References}

Arivazhagan S, Deivalakshmi S, Kannan K, Gajbhiye BN, Muralidhar C, Lukose SN, Subramanian MP (2007) Multi-resolution system for artifact removal and edge enhancement in computerized tomography images. Pattern Recogn Lett 28:1769-1780

Black JM, Sapiro G, Marimont DH, Heeger D (1998) Robust anisotropic diffusion. IEEE Trans Image Process 7(3):421-432

Chen B, Hsu EW (2005) Noise removal in magnetic resonance diffusion tensor imaging. Magn Reson Med 54:393-407

Ding Z, Gore JG, Anderson AW (2005) Reduction of noise in diffusion tensor images using anisotropic smoothing. Magn Reson Med 53:485-490

Gao Q, Zhao Y, Lu Y (2008) Despeckling SAR images using stationary wavelet transform combining with directional filter banks. Appl Math Comput 205:517-524

Hatami S, Hosseini R, Kamarei M, Ahmadi H (2005) Wavelet based fingerprint image enhancement. In: IEEE international symposium on circuits systems, vol 5, Kobe, Japan, May 2005, pp 4610-4613

Huang MY, Tseng DC, Liu MSC (2002) Wavelet image enhancement based on Teager energy operator. In: 16th international conference on pattern recognition, vol 2, Quebec, Canada, August 11-15, pp 993-996

Kim S, Jun S, Lee E, Shin J, Paik J (2009) Ringing artifact removal in digital restored images using multi-resolution edge map. Int $\mathbf{J}$ Signal Process Image Process Pattern Recognit 2(4):95-106

Lysaker M, Lundervold A, Tai X-C (2003) Noise removal using fourth-order partial differential equation with applications to medical magnetic resonance images in space and time. IEEE Trans Image Process 12(12):1579-1590

Marziliano P, Dufaux F (2004) Perceptual blur and ringing metrics: application to JPEG2000. Signal Process Image Commun 19:163-172

Nakashizuka M, Aoki K, Nitta T (2004) A simple edge-weighted image enhancement filter using wavelet scale products. In: 47th IEEE international midwest symposium on circuits and systems, Hiroshima, Japan, July 25-28, pp 285-288

Perona P, Malik J (1990) Scale-space and edge detection using anisotropic diffusion. IEEE Trans Pattern Anal Mach Intell 12(7):629-639

Pesquet JC, Krim H, Carfantan H (1996) Time-invariant orthonormal wavelet representations. IEEE Trans Signal Process 44:1964-1970

Pollak I, Willsky AS, Krim H (2000) Image segmentation and edge enhancement with stabilized inverse diffusion equations. IEEE Trans Signal Process 9(2):256-266

Popovici I, Withers WD (2007) Locating edges and removing ringing artifacts in JPEG images by frequency-domain analysis. IEEE Trans Image Process 16:1470-1474

Weickert J (1998) Anisotropic diffusion in image processing, vol 2. Teubner, Stuttgart, pp 184-189

Yu YJ, Acton ST (2002) Speckle reducing anisotropic diffusion. IEEE Trans Image Process 11(11):1260-1270

Zuo BX, Hu XY (2012) Geophysical model enhancement technique based on blind deconvolution. Comput Geosci 49:170-181

Zuo BX, Ming DL, Tian JW (2009) Perceptual ringing metric to evaluate the quality of images restored using blind deconvolution algorithms. Opt Eng 48(3):1-9 